\title{
Synthesis of bio-inspired Ag-Au nanocomposite and its anti-biofilm efficacy
}

\author{
S NEWASE ${ }^{1}$ and A V BANKAR ${ }^{1,2, *}$ \\ ${ }^{1}$ Department of Microbiology, Savitribai Phule Pune University, Pune 411007, India \\ ${ }^{2}$ Department of Microbiology, Waghire College, Saswad, Affiliated to Savitribai Phule Pune University, \\ Pune 412 301, India
}

MS received 7 June 2016; accepted 24 June 2016

\begin{abstract}
In the present study, bio-inspired Ag-Au nanocomposite was synthesized using banana peel extract (BPE) powder. The Ag-Au nanocomposite was characterized using various techniques such as UV-vis spectrophotometry, transmission electron microscopy (TEM) attached with energy dispersive spectroscopy (EDS) and X-ray diffraction (XRD). Efficiency of AuNPs, AgNPs and Ag-Au nanocomposite was tested for their antibacterial activity against Pseudomonas aeruginosa NCIM 2948. The Ag-Au nanocomposite exhibits enhanced antimicrobial activity over its monometallic counterparts. Anti-biofilm activity of AgNPs, AuNPs and Ag-Au nanocomposite against $P$. aeruginosa was evaluated on glass surfaces. The Ag-Au nanocomposite exhibited the highest biofilm reduction (70-80\%) when compared with individual AgNPs and AuNPs. Effect of AuNPs, AgNPs and Ag-Au nanocomposite on biofilm formation was evaluated in 96 wells microtiter plates. The percentage of biofilm inhibition was sharply increased with increasing concentration of AuNPs, AgNPs and Ag-Au composite. However, Au-Ag nanocomposite showed the highest biofilm inhibition when compared with individual AuNPs and AgNPs. This synergistic anti-biofilm activity of Ag-Au nanocomposite has an importance in the development of novel therapeutics against multidrug-resistant bacterial biofilm.
\end{abstract}

Keywords. Nanocomposite; biological method; anti-biofilm; SEM; TEM; XRD.

\section{Introduction}

Silver and gold nanoparticles (Ag and AuNPs) are the most important nanomaterials that have been studied most extensively. They have some unique optical, electrical and biological properties with a variety of applications in catalysis, optics, imaging, microelectronics, drug delivery, biosensing and biodignostics [1-4]. Physical and chemical methods are widely used for preparation of metal nanoparticles. The chemical methods like chemical reduction, electrochemical techniques and photochemical reduction are most extensively used for preparation of nanomaterials [5]. Conventional methods used for preparation of nanoparticles are found to be complex, costly and hazardous to environment. Thus, biological methods for nanoparticles synthesis could be a possible alternative to the conventional methods.

Biological methods are simple, cost-effective, non-toxic and eco-friendly. Biological systems like yeast, fungi, bacteria and plant extract have been used for nanoparticles synthesis [6-8]. A variety of plant materials such as leaf extract [9], latex [10], fruit extract [11], tuber extract [12], bark powder [13] and banana peel extract (BPE) [14] have been reported

\footnotetext{
*Author for correspondence (ashok@ unipune.ac.in, ashokbankar@gmail.com)
}

earlier for rapid synthesis of metal nanoparticles. Biomaterials are being used for synthesis of bimetallic nanoparticles. Bimetallic core-shell nanoparticles of $\mathrm{Au}$ and $\mathrm{Ag}$ were synthesized using a broth of Neem leaves (Azadirachta indica) [15]. Such nanomaterials have received a special attention due to the possibility of tuning the optical and electronic properties over a broad range. There are rare reports available on biosynthesis of nanocomposites [16]. In the present study, BPE was used as a green source for synthesis of $\mathrm{Ag}-\mathrm{Au}$ nanocomposite. Banana peels are composed of natural polymers and found to be abundant [17], cheap, nontoxic and eco-friendly materials most useful in preparation of nanostructures [14].

\section{Materials and methods}

\subsection{Banana peel extract powder preparation}

Banana peel extract (BPE) powder was prepared by modifying the methodology used in the previous report [14]. The method is briefly described as follows: fresh banana peels (Musa paradisiaca) were collected and thoroughly washed with distilled water. Washed banana peels $(300 \mathrm{~g})$ were crushed into $300 \mathrm{ml}$ of distilled water and kept in boiling water bath for $20 \mathrm{~min}$ at $100^{\circ} \mathrm{C}$. The BPE was filtered and 
the filtrate was precipitated at $4^{\circ} \mathrm{C}$ by adding double volume of chilled acetone. The precipitate was separated by centrifugation at 10,000 rpm for $30 \mathrm{~min}$ and air-dried to obtain dry powder for further experiments.

\subsection{Synthesis of Ag-Au nanocomposites}

AgNPs or AuNPs were synthesized by mixing $2.5 \mathrm{ml}$ of gold chloride or silver nitrate solution of $4 \mathrm{mM}$ with addition of $25 \mathrm{mg}$ BPE powder. Ag-Au nanocomopsite was synthesized by mixing of silver nitrate solution $(8 \mathrm{mM})$ with addition of $50 \mathrm{mg}$ of BPE. The reaction mixture was kept in boiling water bath at $80^{\circ} \mathrm{C}$ for $10 \mathrm{~min}$ and further, $2.5 \mathrm{ml}$ of gold chloride solution $(8 \mathrm{mM})$ was added into the reaction mixture and kept in a boiling water bath at $80^{\circ} \mathrm{C}$ for $10 \mathrm{~min}$.

\subsection{Characterization of nanostructures}

The AuNPs, AgNPs and Au-Ag nanocomposite were characterized by various techniques like UV-vis spectrophotometry (Schimadzu UV spectrophotometer, model UV-1800), transmission electron microscopy (TEM) attached with energy dispersive spectroscopy (EDS) and X-ray diffraction (XRD).

\subsection{Maintenance of bacterial culture}

A wild-type strain of $P$. aeruginosa NCIM 2948 was grown on solid nutrient agar slants at $30^{\circ} \mathrm{C}$ for $24 \mathrm{~h}$. All slants were stored at $4^{\circ} \mathrm{C}$ and the culture was transferred to a new fresh nutrient agar slant after 1 month interval.

\subsection{Synergistic antibacterial activity $\mathrm{Ag}-\mathrm{Au}$ nanocomposite}

Antibacterial activities of $\mathrm{Au}, \mathrm{Ag}$ and $\mathrm{Ag}-\mathrm{Au}$ nanocomposite were tested against $P$. aeruginosa NCIM 2948 by the well diffusion method. A fresh grown culture $(100 \mu \mathrm{l})$ was spread on the sterile nutrient agar plates and standard wells were prepared. All wells were inoculated with $50 \mu$ l of AuNPs, $\mathrm{AgNPs}$ and $\mathrm{Ag}-\mathrm{Au}$ nanocomposite. All plates were incubated at $37^{\circ} \mathrm{C}$ for $24 \mathrm{~h}$ and zone of growth inhibition was observed and measured.

\subsection{Biofilm formation study on glass surface}

Biofilm formation on glass slide surface by $P$. aeruginosa NCIM 2948 was evaluated. A fresh culture of $P$. aeruginosa inoculated into $20 \mathrm{ml}$ of sterile nutrient broth (without AuNPs, AgNPs and Ag-Au nanocomposite) was considered as the control. In the test, a fresh culture was inoculated into three different media containing $20 \mathrm{ml}$ of sterile nutrient broth added with AgNPs, AuNPs and Ag-Au nanocomposite $(0.5 \mathrm{mM})$ separately. Further, the media were poured into separate sterile petriplates containing a glass slide. Biofilm formation or inhibition was visualized and analysed by fluorescence microscopy. For fluorescence microscopy, biofilms were stained with $0.01 \%$ of acridine orange.

\subsection{Effect of AuNPs, AgNPs and Ag-Au nanocomposite on biofilm formation}

A test culture of $P$. aeruginosa NCIM 2948 was inoculated into liquid nutrient broth medium and incubated at $37^{\circ} \mathrm{C}$ for $24 \mathrm{~h}$ on a rotary shaker $(120 \mathrm{rpm})$. The fresh grown culture was adjusted to $\mathrm{OD}_{590}=0.50$. Further, $1 \mathrm{ml}$ of fresh test culture was inoculated into $15 \mathrm{ml}$ of sterile liquid nutrient broth adjusted to different final concentrations $(0.5,1$ and $2 \mathrm{mM}$ ) each of AuNPs, AgNPs and Ag-Au nanocomposite and mixed thoroughly. After proper mixing, $200 \mu \mathrm{l}$ of each medium from respective flasks was added into 96 wells of flat bottomed polysterene microtiter plates. The plates were incubated at $30^{\circ} \mathrm{C}$ for $6,12,18,24$ and $48 \mathrm{~h}$. Plates without nanoparticles or nanocomposite are considered as the control. The biofilm formation by P. aeruginosa NCIM 2948 in the presence or absence of nanoparticles was further quantified by the crystal violet assay method [18]. The methodology is briefly described below. The supernatant along with planktonic cells was discarded. The wells were gently washed with PBS buffer twice and stained with $100 \mu \mathrm{l}$ of crystal violet solution ( $0.3 \%$ in methanol). All plates were incubated at $37^{\circ} \mathrm{C}$ for $5 \mathrm{~min}$. Crystal violet was discarded and washed twice with PBS buffer. Further, $150 \mu$ l of ethanol (95\%) was added into each well and $100 \mu$ solution was transferred into new 96-well microtiter plates. Absorbance of the solution was measured at $595 \mathrm{~nm}$.

\subsection{Statistical analysis}

All experiments were carried out in triplicate. The arithmetic mean was considered for data analysis. Standard deviation and error bars are shown whenever necessary. The unpaired ' $t$ ' test was performed for data comparison. A probability level of $p$ (0.05) was used throughout the study. All statistical analyses of data were carried out by using the GraphPad InStat [DATASET1.ISD] software.

\section{Results and discussion}

\subsection{Visual observation and $U V-v i s$ spectroscopy}

The rapid formation of AuNPs, AgNPs and Ag-AuNPs was detected by visual observation of reaction mixture. Figure $1 \mathrm{a}-\mathrm{c}$ shows a photo of the tube where the colourless solution of silver nitrate was changed into brown colour. Yellow colour of gold chloride solution was turned into faint pink colour. Formation of orange colour was observed after reaction was carried out with silver nitrate, gold chloride and BPE. The appearance of brown, pink and orange colour of solutions indicates formation of AuNPs, AgNPs and Ag-Au nanocomposite.

The UV-vis spectrometry technique is also used for detection of AuNPs, AgNPs and Ag-Au nanocomposite. Figure 1a shows a strong absorbance peak at $420 \mathrm{~nm}$, which is reported to be specific for AgNPs [14]. Absorption peak appearing at $540 \mathrm{~nm}$ indicates formation of AuNPs [19]. 


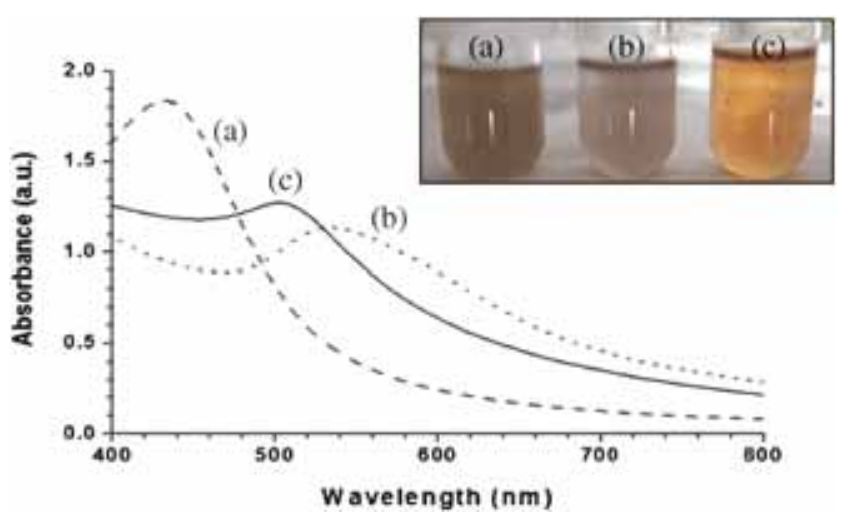

Figure 1. Photographs and UV-vis absorption spectra of solutions after formation of (a) AuNPs, (b) AgNPs and (c) Ag-AuNPs.

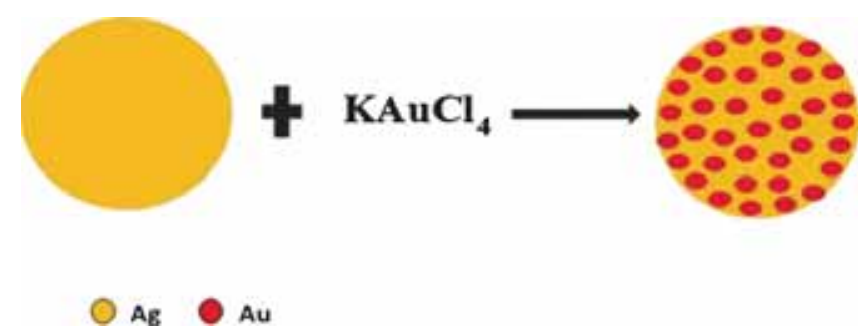

Figure 2. Proposed model demonstrates that $\mathrm{Au}^{3+}$ ions react with $\mathrm{Ag}$ core of the nanoparticle and form an Au shell on AgNPs.

The appearance of a broad absorption peak at $500 \mathrm{~nm}$ is a characteristic of $\mathrm{Ag}-\mathrm{Au}$ nanocomposite. In summary, upon addition of gold chloride solution to the colloidal solution of $\mathrm{AgNPs}$, the $\mathrm{Au}^{3+}$ ions appear to react with the silver core of the nanoparticle and the formation of an Au shell on Ag NPs is schematized in figure 2. A similar $\mathrm{Ag}-\mathrm{Au}$ composite was also reported earlier [20].

\section{$3.2 X$-ray diffraction of nanostructures}

Figure 3 shows that broadening of absorption peaks obtained in the X-ray diffraction (XRD) pattern clearly indicates that particles formed are within the nanometre range. The fcc nature of crystalline nanoparticles was revealed by Bragg's reflections peaks observed. The diffraction peaks for AgNPs observed at $2 \theta=38.03,44.17,65.07$ and $78.02^{\circ}$ correspond to (111), (200), (220) and (311) lattice planes, respectively (JCPDS File No. 04-0783). The XRD patterns of AgNPs are found to be consistent with previous reports [13,14,21]. The diffraction peaks for AuNPs observed at $2 \theta=38.12,44.50$, 64.21 and $77.78^{\circ}$ were assigned to the (111), (200), (220) and (311) lattice planes, respectively (JCPDS File No. 04-0784). The XRD patterns of AuNPs obtained agree well with earlier reports [19,22]. The XRD pattern of Ag-Au nanocomposite clearly revealed that intense peaks appeared at 38.2 and $44.2^{\circ}$ that correspond to (111) and (200) lattice planes, respectively, as compared with other peaks. The XRD pattern

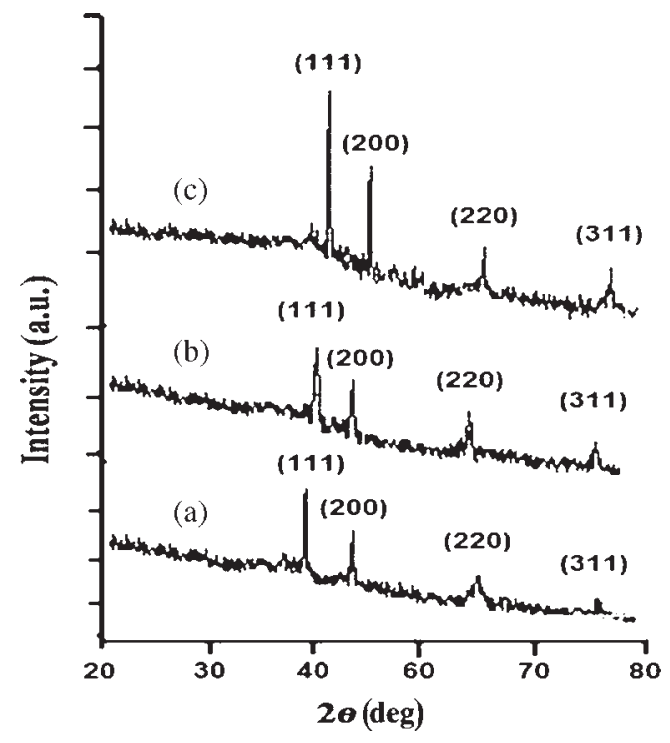

Figure 3. Representative XRD profiles of thin films of (a) AgNPs, (b) AuNPs and (c) $\mathrm{Ag}-\mathrm{Au}$ nanocomposite.

of $\mathrm{Ag}-\mathrm{Au}$ nanocomposite was found to be consistent with a previous report [22].

\subsection{TEM and EDS of nanostructures}

The representative TEM image in figure 4a shows TEM images that depict the presence of spherical AuNPs in the range of 5-20 nm. AgNPs are aggregated into nanoclusters in the range of $20-100 \mathrm{~nm}$ (figure $4 \mathrm{~b}$ ). Figure $4 \mathrm{c}$ and $\mathrm{d}$ reveals that AuNPs formed are assembled onto the surface of the larger AgNPs, thus forming the peculiar core-shell structures. Similar biogenic nanostructures were obtained in previous studies [22,23]. Figure 5 shows the EDS profile of the composite and reflects the presence of specific peaks assigned for AgNPs and AuNPs. This result confirmed the presence of AgNPs and AuNPs in the nanocomposite.

\subsection{Antimicrobial activity of nanostructures}

In recent times, nanostructures have received considerable attention in overcoming the problem of drug resistance developed in pathogens. Several metals are well known for their superior antimicrobial activity [24]. In this regards, antibacterial activity of AuNPs, AgNPs and Au-Ag composites were tested against the pathogenic strain of $P$. aeruginosa NCIM 2948. It was observed that AgNPs and AuNPs exhibit zone diameter of $1.2 \pm 0.05$ and $2.1 \pm 0.05 \mathrm{~cm}$, respectively (figure 6a and b). Antimicrobial activity of biogenic AgNPs and AuNPs is reported earlier [25,26]. Nanoparticles with positive charge attract towards negative charged bacterial cells and bind to the cell membrane via electrostatic attraction. Nanoparticles alter the cell permeability and may cause cell death. The nanoparticles get easy entry into bacterial cells due to their small size and interfere with their DNA, RNA and protein synthesis [27,28]. 

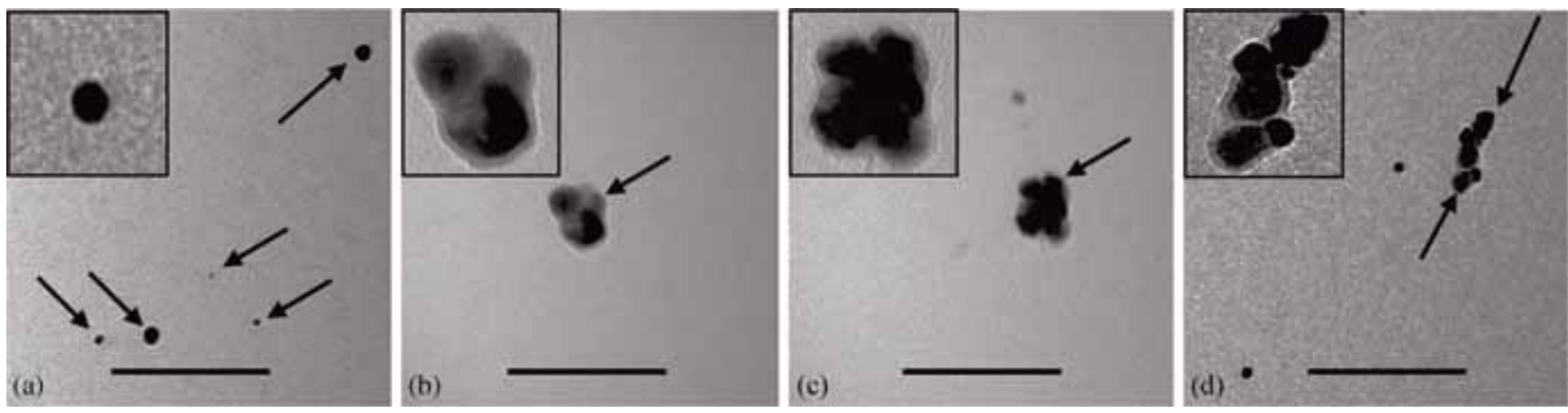

Figure 4. Transmission electron micrographs (TEM) of (a) AuNPs, (b) AgNPs and (c) Ag-Au nanocomposite. Inset bar represents 100 nm.

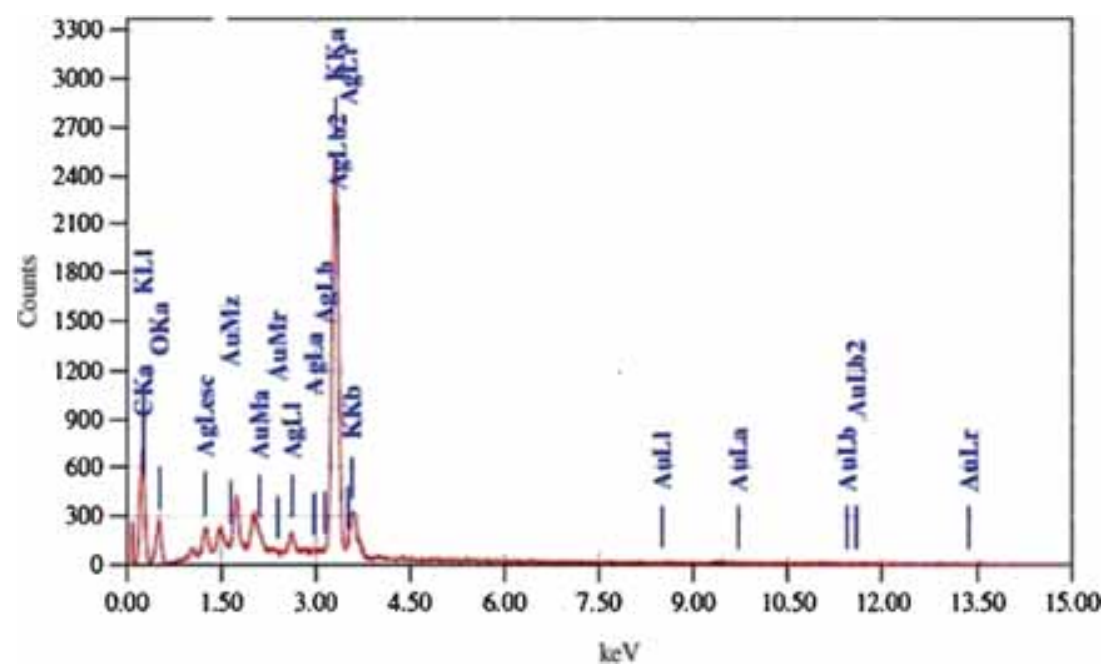

Figure 5. Representative spot EDS profile of $\mathrm{Ag}-\mathrm{Au}$ nanocomposite.

Figure 6c depicts that $\mathrm{Ag}-\mathrm{Au}$ composite showed zone diameter of $3.4 \pm 0.2 \mathrm{~cm}$. The unpaired ' $t$ ' test was used to compare between zone diameters of AuNPs/AgNPs and $\mathrm{Ag}-\mathrm{Au}$ nanocomposite. Such analysis showed that the twotailed $p$-value to be $<0.05$, indicating that the zone diameter difference between $\mathrm{Ag}-\mathrm{Au}$ nanocomposite and AgNPs/ AuNPs was statistically significant. Thus, $\mathrm{Ag}-\mathrm{Au}$ nanocomposite exhibits enhanced antimicrobial activity over its monometallic counterparts. These results are in good agreement with earlier reports $[29,30]$, where enhanced synergistic antibacterial effect of Ag-AuNPs was observed. AgNPs and AuNPs complexes have the ability to competitively intercalate double-stranded genomic DNA [29]. Thus, this study has an importance in the development of a new combination therapy for the treatment of multidrug-resistant pathogens.

\subsection{Anti-biofilm efficacy of Ag-Au nanocomposite on glass surface}

The anti-biofilm efficacy of AuNPs, AgNPs and Ag-Au nanocomposite was evaluated on glass surface. Bacterial biofilms were visualized by fluorescence microscopy as shown in figure 7. A wide range of morphological differences was observed in biofilm architectures due to NPs stress. These results are in agreement with a previous report
[31]. P. aeruginosa exhibits the ability to form biofilm on glass surface without nanoparticles (control). AuNPs and AgNPs have reduced about 25 and $30 \%$ of biofilm formation, respectively. Ag-Au nanocomposite showed biofilm reduction of about $70-80 \%$. The unpaired ' $t$ ' test was used to compare between biofilm reduction (\%) by AuNPs/AgNPs and $\mathrm{Ag}-\mathrm{Au}$ nanocomposite. Analysis revealed the two-tailed $p$-value to be $<0.05$, indicating that biofilm reduction (\%) difference by $\mathrm{Ag}-\mathrm{Au}$ nanocomposite and $\mathrm{AgNPs} / \mathrm{AuNPs}$ was statistically significant.

This clearly indicates that anti-biofilm activity of $\mathrm{Ag}-\mathrm{Au}$ nanocomposite was enhanced when compared with monometallic counterparts. These obtained results are in agreement with an earlier report [29], where $\mathrm{Ag}-\mathrm{Au}$ biometallic exhibited anti-biofilm effect against $P$. aeruginosa.

\subsection{Inhibition of bacterial biofilms by nanoparticles}

The characteristic of biofilm formation by $P$. aeruginosa has an importance in pathogenicity. Figure 8 reflects effect of AuNPs, AgNPs and Ag-Au nanocomposite on biofilm formation by $P$. aeruginosa in wells of microtiter plates. It was seen that AgNPs and AuNPs showed a remarkable reduction in the biofilm formation by $P$. aeruginosa. This might be due to the interactions that occur between bacterial cells 

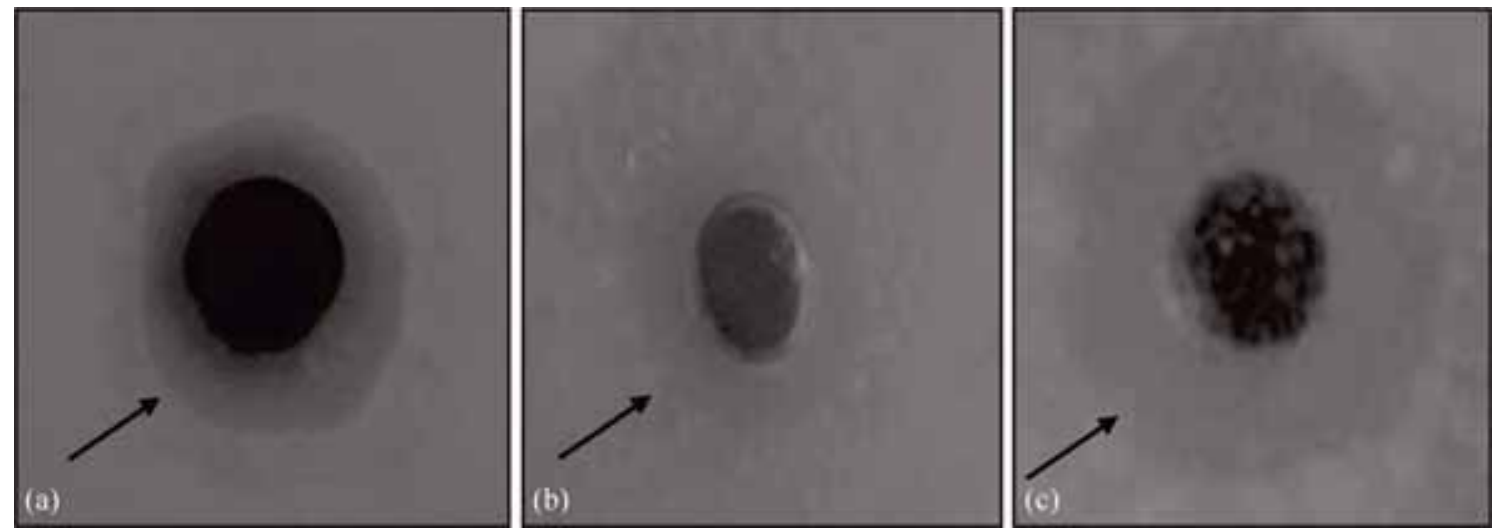

Figure 6. Photograph of growth inhibition of Pseudomonas aeroginosa on nutrient agar plate by (a) AuNPs, (b) AgNPs and (c) Ag-Au nanocomposite.
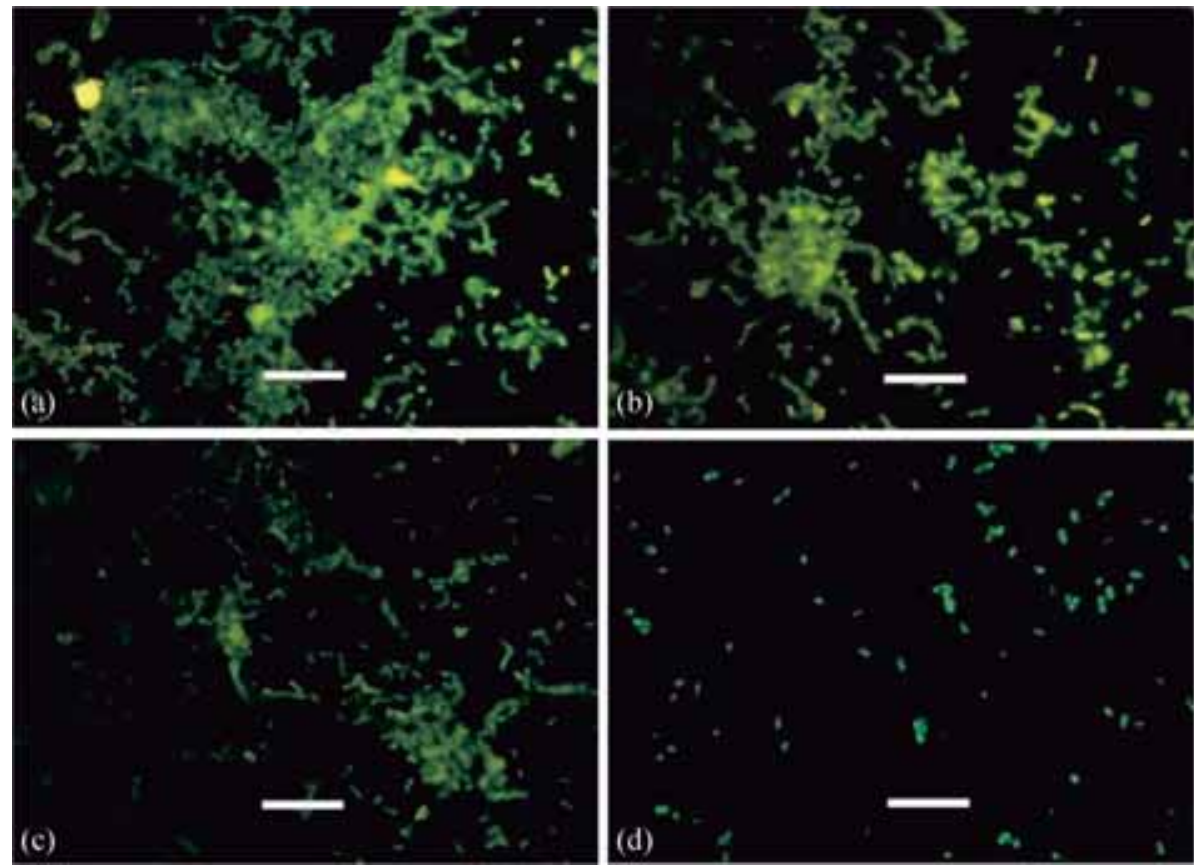

Figure 7. Fluorescence images of (a) biofilm formation by $P$. aeroginosa without NPs (control) and in the presence of (b) AgNPs, (c) AuNPs and (d) Ag-Au nanocomposite. Inset bar represents $10 \mu \mathrm{m}$.
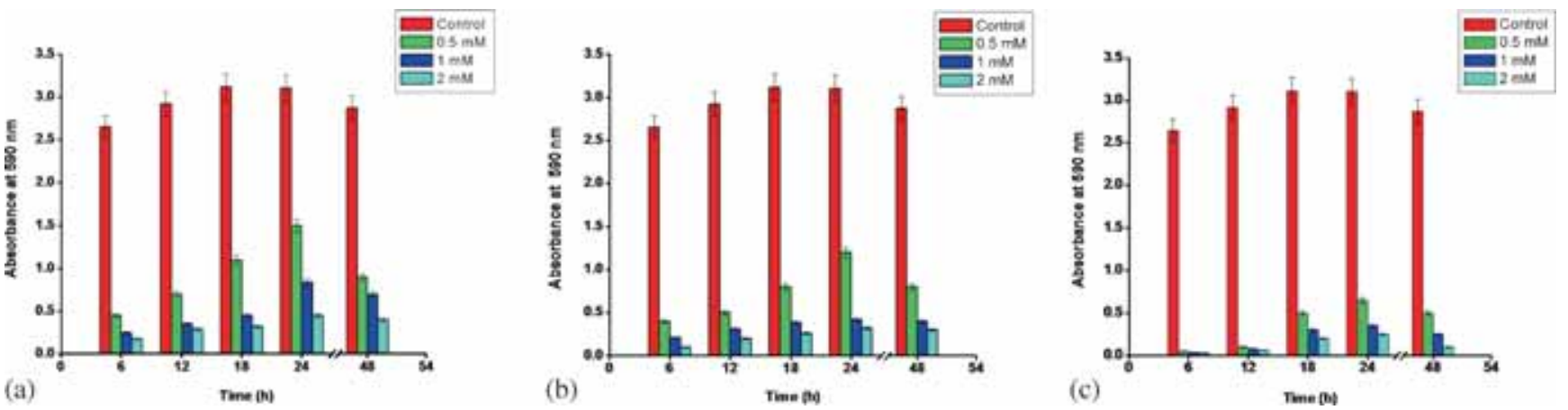

Figure 8. Biofilm formation by P. aeroginosa in 96 wells of microtiter plates (a) in the absence of NPs and in the presence of (b) AgNPs, (c) AuNPs and (d) Ag-Au nanocomposite. 
and nanoparticles. These results are in good agreement with previous reports $[26,32]$. It is seen that biofilm formation without nanoparticles was established after $6 \mathrm{~h}$ and reached the maximum level (maturation) at about $24 \mathrm{~h}$ (control). In the test, biofilm was not well established at $6 \mathrm{~h}$ and showed reduction in biofilm even at $24 \mathrm{~h}$ of incubation. After $24 \mathrm{~h}$ incubation, it was noticed that AgNPs resulted in a significant decrease of $50.17,72.10$ and $80.05 \%$ in biofilm formation at concentration of $0.5,1.0$ and $2.0 \mathrm{mM}$, respectively. It was observed that AuNPs showed biofilm reduction of about 66.12, 81.05 and $85.37 \%$. Similarly, Ag-Au nanocomposite shows biofilm reduction of about $78.41,88.38$ and $91.70 \%$ (figure 8). In all these cases, the percentage of biofilm formation was sharply decreased with increasing concentration of nanoparticles. Thus, AuNPs and AgNPs were effective against the biofilm formation by $P$. aeruginosa. It was also seen that $\mathrm{Ag}-\mathrm{Au}$ nanocomposite showed the maximum biofilm inhibition (\%) against $P$. aeruginosa biofilm, which was statistically significant $(p<0.05)$, when compared with monometallic AuNPs/AgNPs. Thus, synergistic anti-biofilm effect of $\mathrm{Ag}-\mathrm{Au}$ nanocomposite was observed. It has been reported that this result might be due to destabilization of bacterial biofilms [33].

\section{Conclusion}

In the present study, the biological method used for synthesizing $\mathrm{Ag}-\mathrm{Au}$ nanocomposite has a distinct advantage over chemical methods such as high efficiency, cost-effectiveness, easy operation, eco-friendliness and non-toxicity to environment. The $\mathrm{Ag}-\mathrm{Au}$ nanocomposite exhibits enhanced antimicrobial and anti-biofilm characteristics over its monometallic counterparts. This reflects its importance in future development of 'therapeutic agents' against the multidrug-resistant bacterial biofilms.

\section{References}

[1] Nair L S and Laurencin C T 2007 J. Biomed. Nanotechnol. 3301

[2] Lee K S and El-Sayed M A 2006 J. Phys. Chem. B 11019220

[3] Jain P K, Huang X, El-Sayed I H and El-Sayed M A 2008 Acc. Chem. Res. 411578

[4] Jennings T and Strouse G 2007 Adv. Exp. Med. Biol. 62034

[5] Daniel M and Astruc D 2004 Chem. Rev. 104293

[6] Kowshik M, Ashtaputre S, Kharrazi S, Vogel W, Urban J, Kulkarni S and Paknikar K 2003 Nanotechnology 1495

[7] Senapati S, Ahmad A, Khan M, Sastry M and Kumar R 2005 Small 1517

[8] Shahverdi A R, Minaeian S, Shahverdi H R, Jamalifar H and Nohi A A 2007 Process Biochem. 42919
[9] Shiv Shankar S, Ahmad A and Sastry M 2003 Biotechnol. Prog. 191627

[10] Bar H, Bhui D K, Sahoo G P, Sarkar P, De S P and Misra A 2009 Colloids Surf. B Biointerfaces 339134

[11] Jain D, Kumar D, Kachhwaha S and Kothari L 2009 Dig. J. Nanomater. Biostruct. 4557

[12] Ghosh S, Patil S, Ahire M, Kitture R, Kale S, Pardesi K, Cameotra S S, Bellare J, Dhavale D D, Jabgunde A and Chopade B A 2012 Int. J. Nanomed. 7483

[13] Sathishkumar M, Sneha K, Won W S, Cho C W, Kim S and Yun Y S 2009 Colloids Surf. B Biointerfaces 73332

[14] Bankar A, Joshi B, Kumar A and Zinjarde S 2010 Colloids Surf. A Physicochem. Eng. Aspects $\mathbf{3 6 8} 58$

[15] Shankar S S, Rai A, Ahmad A and Sastry M 2004 J. Colloid Interface Sci. 275496

[16] Mandal S, Selvakannan P R, Pasricha R and Sastry M 2003 J. Am. Chem. Soc. 1258440

[17] Emaga T H, Robert C, Ronkart S N, Wathelet B and Paquot M 2007 Bioresour. Technol. 994346

[18] Dusane D H, Rajput J K, Kumar A R, Nancharaiah Y V, Venugopalan V P and Zinjarde S S 2008 Lett. Appl. Microbiol. 47374

[19] Bankar A, Joshi B, Kumar A and Zinjarde S 2010 Colloids Surf. B Biointerfaces $\mathbf{8 0} 45$

[20] Sánchez-Muñoz O L, Salgado J, Martínez-Pastor J and Jiménez-Villar E 2012 Nanosci. Nanotechnol. 21

[21] Huang J, Li Q, Sun D, Lu Y, Su Y, Yang X, Wanh H, Wang Y, Shao W, He N, Hong J and Chen C 2007 Nanotechnology 181

[22] Nagaonkar D and Rai M 2015 Adv. Mater. Lett. 6334

[23] Shivshankar S, Rai A, Ahmad A and Sastry M 2004 J. Colloid Interface Sci. 275496

[24] Gong P, Li H, He X, Wang K, Hu J, Tan W, Zhang S and Yang X 2007 Nanotechnology 18604

[25] Ali Z A, Yahya R, Sekaran S D and Puteh R 2016 Adv. Mater. Sci. Eng. (doi: 10.1155/2016/4102196)

[26] Nithya B and Jayachitra A 2016 Int. J. Pure Appl. Biosci. 4201

[27] Dibrov P, Dzioba J, Gosink K K and Hase C C 2002 Antimicrob. Agents Chemother. $\mathbf{4 6} 2668$

[28] Shrivastava S, Tanmay B E, Roy A, Singh G, Rao P R and Dash D 2007 Nanotechnology 181

[29] Savić N D, Milivojevic D R, Glišić B D, Ilic-Tomic T, Veselinovic J, Pavic A, Vasiljevic B, Nikodinovic-Runic J and Djuran M I 2016 RSC Adv. 613193

[30] Ramasamy M, Lee M J and Lee J J 2016 J. Biomater. Appl. 31366

[31] Mu H, Tang J, Liu Q, Sun C, Wang T and Duan J 2016 Sci. Rep. 618877

[32] Barapatre A, Aadil K R and Jha H 2016 Bioresour. Bioprocess. 38

[33] Chaw K C, Manimaran M and Tay F E H 2005 Antimicrob. Agents Chemother. 494853 